\title{
Robustness of IFDMA as Air Interface Candidate for Future High Rate Mobile Radio Systems
}

\author{
T. Frank ${ }^{1}$, A. Klein ${ }^{1}$, E. Costa ${ }^{2}$, and E. Schulz ${ }^{2}$ \\ ${ }^{1}$ TU Darmstadt, Institut für Nachrichtentechnik, Merckstr. 25, 64283 Darmstadt, Germany \\ ${ }^{2}$ Siemens AG, Sankt-Martin-Str. 76, 81541 München, Germany
}

\begin{abstract}
The Interleaved Frequency Division Multiple Access (IFDMA) scheme is a candidate for next generation mobile radio systems. IFDMA is based on compression, repetition and subsequent user dependent frequency shift of a modulated signal. As in OFDMA, multiple access is enabled by the assignment of overlapping but mutually orthogonal subcarriers to each user. It provides various advantages such as low peak to average power ratio, orthogonality of the signals of different users even for transmission over a time dispersive channel and low complexity. However, IFDMA is sensitive to frequency offsets. In this paper, a derivation of IFDMA based on a generalised OFDMA signal model with specific precoding and specific subcarrier allocation is presented. Moreover, simulation results showing the robustness properties of IFDMA against frequency errors are given.
\end{abstract}

\section{Introduction}

Presently, research for beyond 3rd generation mobile radio systems is in progress world wide. A future mobile radio system has to meet challenging requirements. On the one hand it should enable different types of services from data rates of a few kbit/s up to several Mbit/s. Moreover, it should provide high flexibility and granularity as well as high performance. On the other hand, low cost and hence, low complexity implementation is requested, especially for mobile terminals.

An important decision for a future mobile radio system is the choice of the multiple access scheme it is based on. One possible candidate is OFDMA, where a number of overlapping but mutually orthogonal subcarriers is assigned to each user (Dinis et al., 2004). A basic advantage of OFDMA is that even for transmission over time-dispersive channels the signals of different users remain orthogonal if perfect carrier synchronisation is assumed. However, OFDMA signals are sensitive to frequency offsets due to, e.g., Doppler effects or oscillator imperfections. Moreover, they have a high peak to average power ratio and hence, lead to inefficient use of the power amplifier.

Correspondence to: T. Frank

(t.frank@nt.tu-darmstadt.de)
Another possible solution is DS-CDMA which is based on spreading the signals of different users in frequency domain by the use of spreading sequences which are orthogonal to each other (Dinis et al., 2004). In general, CDMA signals provide low envelope fluctuations. However, the orthogonality of different users' signals is affected, if CDMA signals are transmitted over a time-dispersive channel. Hence, for CDMA computational complexity for user separation can be high.

A further promising candidate for a future mobile radio system is Interleaved Frequency Division Multiple Access (IFDMA) (Sorger et al., 1998). It is based on compression, repetition and subsequent user dependent frequency shift of a modulated signal. As in OFDMA, multiple access is enabled by the assignment of overlapping but mutual orthogonal subcarriers to each user. It combines the advantages of spread-spectrum signals such as, e.g., DS-CDMA, and multi carrier solutions such as, e.g., OFDMA i.e., it provides a low peak to average power ratio as well as low complexity for user separation, because also for IFDMA the orthogonality of the signals is not affected by transmission over a timedispersive channel (Sorger et al., 1998). However, IFDMA is closely related to OFDMA and hence, it is also expected to be sensitive to frequency offsets. Therefore, in this paper IFDMA is derived based on a generalised signal model for precoded OFDMA and robustness properties of IFDMA are investigated by simulation in order to show the effect of frequency errors on IFDMA.

The paper is organized as follows. In Sect. 2, signal generation and the most important signal properties of IFDMA are described. In Sect. 3, it is shown how multiple access is enabled in IFDMA. A derivation of IFDMA based on a generalised OFDMA signal model is given in Sect. 4. Finally, in Sect. 5, some simulation results for IFDMA are presented which show the effect of frequency errors.

\section{IFDMA signal generation}

In this section, signal generation and signal properties of a time continuous IFDMA signal for one user are presented. The user is indicated by index $k, k=0, \cdots, K-1$, where $K$ 


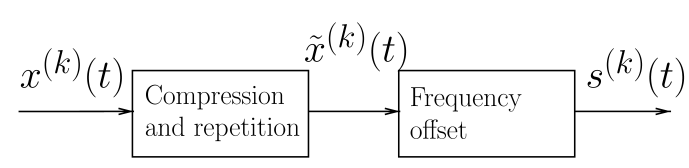

Fig. 1. Continuous Time IFDMA Signal Model.

designates the total number of users in a system. A data block of the $k$-th user containing $Q$ symbols is described by

$\mathbf{d}^{(k)}=\left(d_{1}^{(k)}, \cdots, d_{Q}^{(k)}\right)^{T}, \quad k=0, \cdots, K-1$.

According to Sorger et al. (1998) for example, an IFDMA signal can be generated by compression and periodic repetition in time domain of a modulated signal and subsequent frequency shift to the desired position of the signal in frequency domain. A continuous time IFDMA signal model for the $k$-th user is depicted in Fig. 1 where $x^{(k)}(t)$ designates the modulated signal, $\tilde{x}^{(k)}(t)$ designates the compressed and repeated signal and $s^{(k)}(t)$ designates the IFDMA signal after frequency shift. Furthermore, let $f_{s}(t)$ designate the pulseshape filter and let $T_{s}$ designate the symbol length. In case of linear modulation, the modulated and pulse-shaped continuous time signal $x^{(k)}(t)$ of user $k$ can be described by

$x^{(k)}(t)=\sum_{q=1}^{Q} d_{q}^{(k)} \cdot f_{s}\left(t-q T_{s}\right), \quad k=0, \cdots, K-1$,

where $d_{q}^{(k)}$ represents the $q$-th element of the symbol vector given by Eq. (1). Let the integer $L$ designate the compression factor and the number of repetitions, respectively, and let $\tilde{f}_{s}(t)$ designate the modified pulse shape filter according to

$\tilde{f}_{s}(t)=f_{s}(L t)$.

Thus, compression and repetition of signal $x^{(k)}(t)$ can be described by

$\tilde{x}^{(k)}(t)=\sum_{l=0}^{L-1} \sum_{q=1}^{Q} d_{q}^{(k)} \cdot f_{s}\left(L t-q T_{s}-l Q T_{s}\right)=$

$\sum_{\mu=1}^{L Q} d_{\mu \bmod Q} \cdot \tilde{f}_{S}\left(t-\mu T_{c}\right)$,

where $T_{c}$ designates the chip length according to

$T_{c}=\frac{T_{s}}{L}$.

Examples for the input signal $x^{(k)}(t)$, the compressed signal $x^{(k)}(L t)$ and the repeated signal $\tilde{x}^{(k)}(t)$ for $L=3$ are given in Fig. 2. Let signal $x^{(k)}(t)$ be limited to length $T$ according to

$T=Q \cdot T_{s}$.
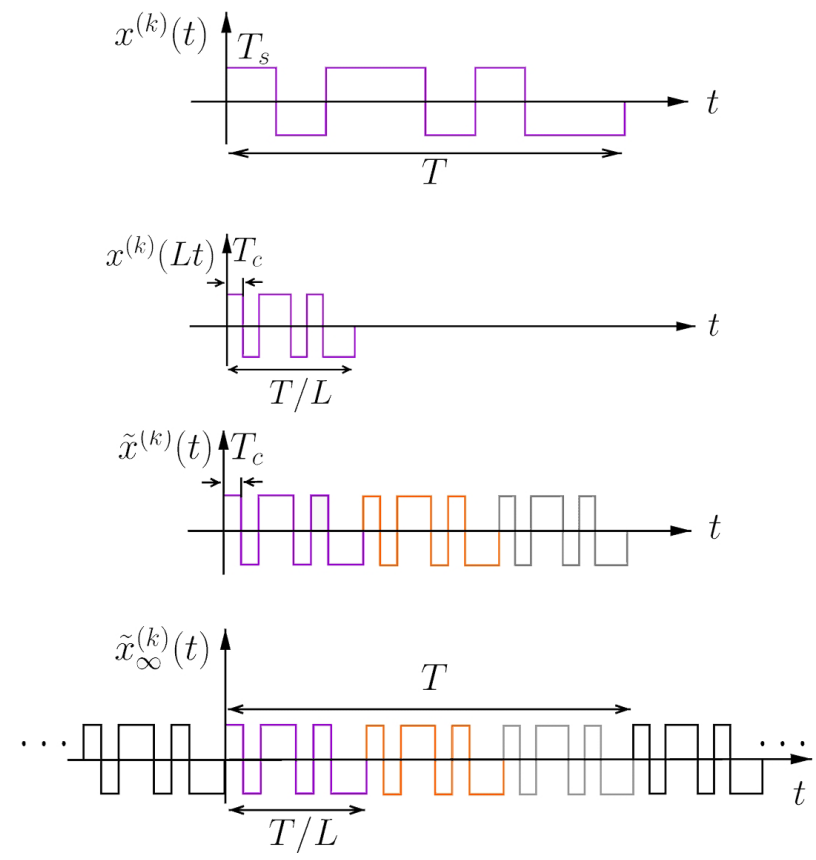

Fig. 2. Example, IFDMA signal generation, $L=3, Q=8$.

Thus, the compressed and repeated signal $\tilde{x}^{(k)}(t)$ given by Eq. (4) can be represented by windowing of an infinite periodic signal $\tilde{x}_{\infty}^{(k)}(t)$ as depicted in Fig. 2 given by

$\tilde{x}_{\infty}^{(k)}(t)=\sum_{l=-\infty}^{\infty} \sum_{q=1}^{Q} d_{q}^{(k)} \cdot f_{s}\left(L t-q T_{s}-l Q T_{s}\right)$

using a window function according to

$w(t)=\operatorname{rect}\left(\frac{t-T / 2}{T}\right)$,

i.e.,

$\tilde{x}^{(k)}(t)=w(t) \cdot \tilde{x}_{\infty}^{(k)}(t)$.

Let $W(f)$ and $\tilde{X}_{\infty}^{(k)}(f)$ designate the Fourier transforms of $w(t)$ and $\tilde{x}_{\infty}^{(k)}(t)$, respectively. For a rectangular window function $w(t)$ according to Eq. (8), $W(f)$ results in a sifunction with zero points in a distance of

$\Delta f_{z}=\frac{1}{T}$.

The periodic function $\tilde{x}_{\infty}^{(k)}(t)$ with period $T / L$ can be represented by a Fourier-series and, therefore, has a discrete spectrum with spectral lines at a distance of

$\Delta f_{s}=\frac{L}{T}$.

Thus, in frequency domain, Eq. (9) can be described by

$\tilde{X}^{(k)}(f)=W(f) * \tilde{X}_{\infty}^{(k)}(f)=K \cdot \operatorname{si}\left(\frac{\pi f}{T}\right) * \tilde{X}_{\infty}^{(k)}(f)$. 

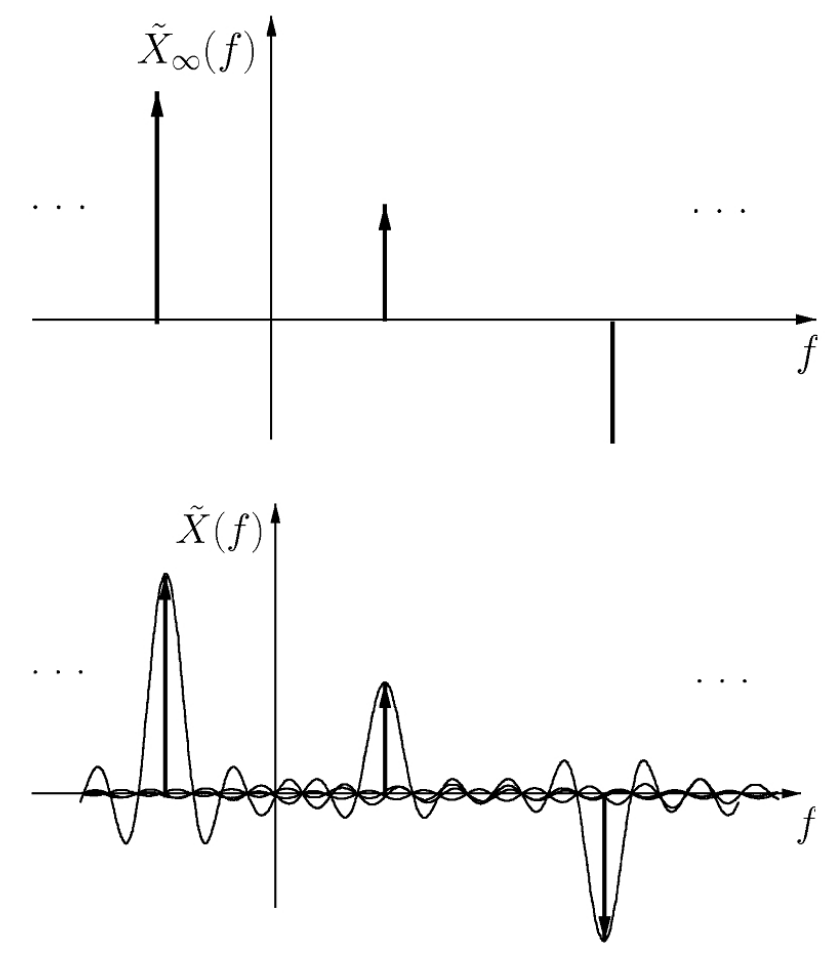

Fig. 3. Illustration of convolution in Eq. (12) for $L=8$.

where $K$ designates a complex constant, $\operatorname{si}(x)=\sin (x) / x$ and * designates linear convolution. Due to convolution with $W(f)$, the spectral lines of $\tilde{X}_{\infty}^{(k)}(f)$ result in the local maxima of $\tilde{X}^{(k)}(f)$. In the following, these local maxima are designated as subcarriers of signal $\tilde{x}^{(k)}(t)$. Together with Eq. (10), the distance $\Delta f_{s}$ of the spectral lines of $\tilde{X}_{\infty}^{(k)}(f)$ or of the subcarriers of $\tilde{x}^{(k)}(t)$, respectively, can be written as

$\Delta f_{s}=L \cdot \Delta f_{z}$

From Eqs. (10) and (13) follows that the spectrum $\tilde{X}^{(k)}(f)$ of the compressed and repeated signal $\tilde{x}^{(k)}(t)$ has exactly $L-1$ zero points inbetween two arbitrarily chosen subcarriers. The convolution described by Eq. (12) is schematically shown in Fig. 3. Examples for the absolute value of the spectrum $\tilde{X}^{(k)}(f)$ of an IFDMA-signal are given in Fig. 4. With Eq. (5) the total bandwidth of an IFDMA-signal is given by

$B=\frac{1}{T_{c}}$

Using Eqs. (5) and (6), from Eq. (14) follows

$B=Q \cdot \frac{L}{T}$.

Hence, together with the subcarrier distance given in Eq. (11), Eq. (15) shows that an IFDMA signal for one user has exactly $Q$ equidistant subcarriers within the total bandwidth $B$. Each subcarrier contains information about all symbols of vector $\mathbf{d}^{(k)}$.

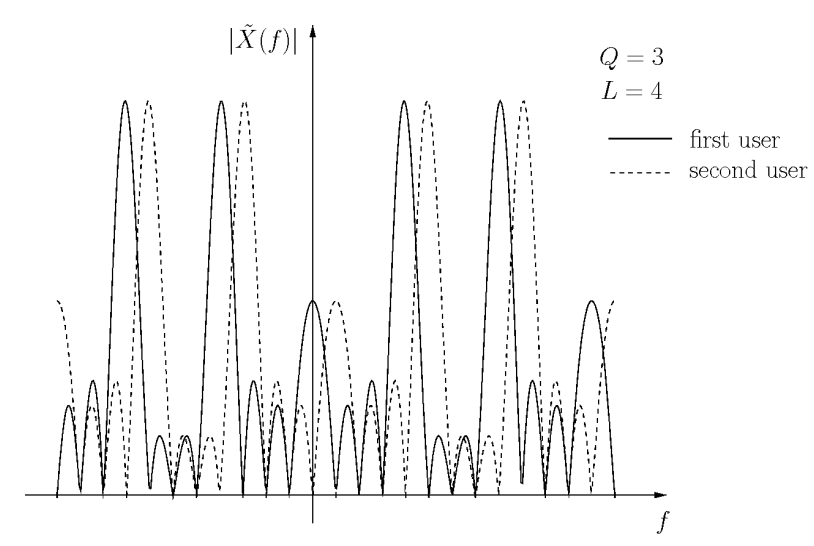

Fig. 4. Absolute value of the spectrum of an IFDMA signal for 2 users.

\section{Multiple-access}

In the following, it is shown how multiple-access can be realized for IFDMA. As mentioned in Sect. 2, the IFDMA signal of each user has exactly $L-1$ equidistant zero points inbetween two arbitrarily chosen adjacent subcarriers of its spectrum. Hence, at the zero points, $L-1$ signals of other users can be positioned by appropriate frequency shift in order to enable frequency-division multiple-access (FDMA). Thus, the compression factor $L$ is closely related to the maximum number of users $K$ that can be served in a system and an appropriate choice for $L$ might be

$L=K$.

It can be proven that for $K \leq L$ the signals of all users are orthogonal to each other and that orthogonality is not affected even for transmission over a time invariant multi-path channel (Sorger et al., 1998). Positioning the signals of different users in the zero points of the other users is realized by a user specific frequency shift according to

$\Phi_{k}=k \frac{2 \pi}{T}=k \frac{2 \pi}{Q L T_{c}}$,

as is illustrated in Fig. 4 for two users. Hence, the IFDMA signal of multiple users can be described by

$s(t)=\sum_{k=0}^{K-1} s^{(k)}(t)=\sum_{k=0}^{K-1} \tilde{x}^{(k)}(t) \cdot e^{-j \Phi_{k} t}$,

cf. Fig. 1.

\section{Generalised Signal Model}

The IFDMA-scheme can also be derived based on a generalised discrete-time signal model for precoded OFDMA in the equivalent low-pass domain as it is depicted in Fig. 5. We consider one user with index $k$ transmitting the data vector $\mathbf{d}^{(k)}$ according to Eq. (1). According to the signal model in Fig. 5, after serial-to-parallel conversion, firstly, a linear 


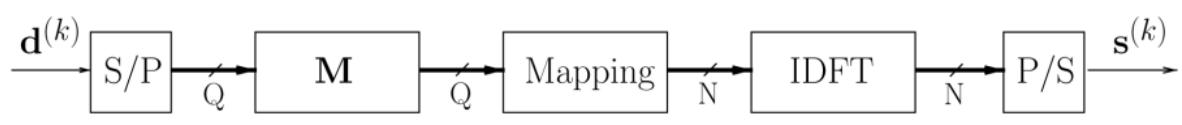

Fig. 5. Generalised signal model for an OFDMA system.

operation described by the $(Q \times Q)$-matrix $\mathbf{M}$ is applied to the input vector $\mathbf{d}^{(k)}$ where matrix $\mathbf{M}$ can be interpreted as a linear precoding matrix (Xia et al., 2003). Let $N$ designate the total number of subcarriers available in an OFDMA system and let $Q$ designate the number of subcarriers assigned to each user. The total number of subcarriers shall be assumed as

$N=K \cdot Q$,

where $K$ designates the number of users. In order to avoid inter user interference, the $Q$ subcarriers of each user have to be mapped to the $N$ subcarriers available in the system in such a way that subcarriers of different users do not overlap. The assignment of the precoded data symbols after mapping to the subcarriers can be described by an $N \times N$ Inverse Discrete Fourier Transform (IDFT) described by matrix IDFT $_{N}$ according to

$\operatorname{IDFT}_{N}=$
$\frac{1}{\sqrt{N}}\left(\begin{array}{cccc}1 & 1 & \cdots & 1 \\ 1 & e^{j \frac{2 \pi}{N}(1)} & \cdots & e^{j \frac{2 \pi}{N}(N-1)} \\ \vdots & \vdots & \ddots & \vdots \\ 1 e^{j \frac{2 \pi}{N}(N-1)} & \cdots e^{j \frac{2 \pi}{N}(N-1)(N-1)}\end{array}\right)$.

The output vector of the OFDMA system after parallel-toserial conversion is represented by $\mathbf{s}^{(k)}$,

$\mathbf{s}^{(k)}=\left(s_{1}^{(k)}, \cdots, s_{Q}^{(k)}\right)^{T}, \quad k=0, \cdots, K-1$.

If the matrix $\mathbf{M}$ is chosen according to

$\mathbf{M}=\mathbf{I}$

where $\mathbf{I}$ designates the $(Q \times Q)$-identity matrix, the output vector $\mathbf{s}^{(k)}$ represents an uncoded OFDMA signal. For a unitary matrix $\mathbf{M}$, the output vector represents a unitary precoded OFDM (UP-OFDM) signal as it is described, e.g., in Xia et al. (2003). One possible choice for a unitary matrix $\mathbf{M}$ is given by

$\mathbf{M}=\mathbf{D F T}_{Q}$,

where DFT $_{Q}$ designates the $Q$-point Discrete Fourier Transform (DFT) matrix

DFT $_{Q}=$

$\frac{1}{\sqrt{Q}}\left(\begin{array}{cccc}1 & 1 & \cdots & 1 \\ 1 & e^{-j \frac{2 \pi}{Q}(1)} & \cdots & e^{-j \frac{2 \pi}{Q}(Q-1)} \\ \vdots & \vdots & \ddots & \vdots \\ 1 e^{-j \frac{2 \pi}{Q}(Q-1)} & \cdots & e^{-j \frac{2 \pi}{Q}(Q-1)(Q-1)}\end{array}\right)$.
In this case, the generalised signal model depicted in Fig. 5 is equal to a structure described by Galda et al. (2002). Let us assume the $Q$ subcarriers of each user $k, k=0, \ldots, K-1$, to be equidistantly allocated and equally distributed over the whole bandwidth. Allocating the $Q$ subcarriers of one user equidistantly over the total bandwidth is equal to the insertion of $K-1$ subcarriers modulated by zero inbetween two adjacent subcarriers modulated by elements of the precoded input vector $\mathbf{M} \cdot \mathbf{d}^{(k)}$. The insertion of zeros combined with the precoding matrix $\mathbf{M}$ can be expressed by

$$
\begin{aligned}
& \widehat{\mathbf{M}}=\mathbf{D F T}_{N} \cdot \mathbf{R}= \\
& \frac{1}{\sqrt{N}}\left(\begin{array}{cccc}
1 & 1 & \cdots & 1 \\
1 & e^{-j \frac{2 \pi}{N}(1)} & \cdots & e^{-j \frac{2 \pi}{N}(N-1)} \\
\vdots & \vdots & \ddots & \vdots \\
1 & e^{-j \frac{2 \pi}{N}(N-1)} & \cdots & e^{-j \frac{2 \pi}{N}(N-1)(N-1)}
\end{array}\right) . \\
& \underbrace{\left(\begin{array}{cccc}
1 & 0 & \cdots & 0 \\
0 & 1 & \cdots & 0 \\
\vdots & \vdots & \ddots & \vdots \\
0 & 0 & \cdots & 1 \\
\vdots & \vdots & \vdots & \vdots \\
1 & 0 & \cdots & 0 \\
0 & 1 & \cdots & 0 \\
\vdots & \vdots & \ddots & \vdots \\
0 & 0 & \cdots & 1
\end{array}\right)}_{Q}\} Q\} N=K \cdot Q,
\end{aligned}
$$

where $\mathbf{D F T}_{N}$ designates the $N$-point DFT and $\mathbf{R}$ designates a $(N \times Q)$ repetition matrix. In order to avoid multiple access interference, let us further assume that the subcarrier set of user $k$ has a frequency offset of

$\Phi^{(k)}=\frac{2 \pi}{N} k$.

In this case, the generalised OFDMA scheme in Fig. 5 can be described by

$$
\mathbf{s}^{(k)}=\boldsymbol{\Phi}^{(k)} \cdot \mathbf{I D F T}_{N} \cdot \widehat{\mathbf{M}} \cdot \mathbf{d}^{(k)}=\boldsymbol{\Phi}^{(k)} \cdot \mathbf{I D F T}_{N} \cdot \mathbf{D F T}_{N} \cdot \mathbf{R} \cdot \mathbf{d}^{(k)},
$$

where $\boldsymbol{\Phi}^{(k)}$ designates the frequency shift operation by rotation with $e^{j \Phi^{(k)} n}, n=1, \ldots, N-1$ in time domain and is given by the $(N \times N)$-matrix

$\boldsymbol{\Phi}^{(k)}=\left(\begin{array}{ccc}e^{j \frac{2 \pi}{N} k \cdot 0} & \cdots & 0 \\ \vdots & \ddots & \vdots \\ 0 & \cdots & e^{j \frac{2 \pi}{N} k(N-1)}\end{array}\right)$. 

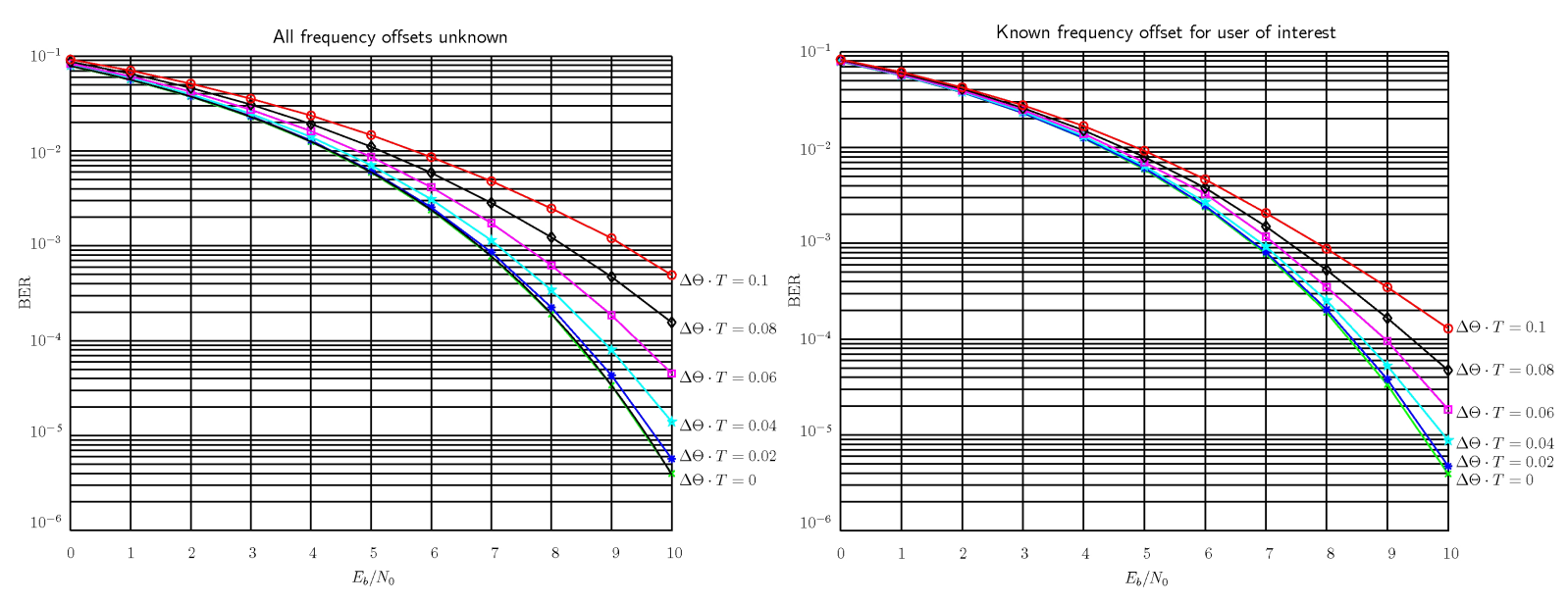

Fig. 6. Simulation results without and with knowledge of the frequency error for user of interest at the receiver.

Table 1. Simulation parameters (AWGN channel).

\begin{tabular}{llll}
\hline Compression/repetition factor: & $K=L=16$ & Subcarrier spacing: & $1 / T=39 \mathrm{kHz}$ \\
Number of symbols: & $Q=64$ & Relative frequency offset: & $\Delta \Theta \cdot T=0,0.02, \ldots, 0.1$ \\
Total number of subcarriers: & $N=1024$ & Bandwidth: & $B=40 \mathrm{MHz}$ \\
\hline
\end{tabular}

Hence, using Eq. (27) an OFDMA signal with DFT precoding and equidistantly distributed subcarriers and frequency offset according to Eq. (28) for each user can be written as

$$
\mathbf{s}^{(k)}=\boldsymbol{\Phi}^{(k)} \cdot \mathbf{R} \cdot \mathbf{d}^{(k)}
$$

which is equal to a discrete time equivalent of the signal model described by Fig. 1.

Another approach in order to derive IFDMA is based on an alternative CDMA scheme using Frequency Domain Orthogonal Spreading Sequences (FDOSS) as it is described, e.g., in Chang and Chen (2000). Moreover, IFDMA can be combined with conventional CDMA schemes as described, e.g., in Goto et al. (2003).

\section{Simulation results}

As shown in Sect. 4, IFDMA can be described by a precoded OFDMA scheme with subcarriers equidistantly allocated over the whole bandwidth. Hence, it can be expected that similar to OFDM, IFDMA is sensitive to frequency offsets. Therefore, in the following the sensitivity of a fully loaded IFDMA-system are investigated by simulation for transmission over an additional white Gaussian noise (AWGN) channel in an up-link scenario. We consider frequency errors due to oscillator inaccuracies and Doppler shifts. Let $\Delta \Theta_{\max }$ designate the maximum frequency error. For each user, the frequency error is described by a random variable $\Delta \Theta$ which is assumed to be uniformly distributed in
$\left[-\Delta \Theta_{\max } ; \Delta \Theta_{\max }\right]$. The simulation parameters are given in table 1 . The influence of frequency offsets to the demodulation process can be described by two effects. On the one hand orthogonality between the signals of different users is lost, because the signals are not positioned in the zero points of the other ones. Hence, we have multiple access interference (MAI). On the other hand, for demodulation of the received signal of the $k$-th user, at the receiver, power of the useful signal is lost, because the subcarrier frequencies at the receiver do not meet the local maxima of the useful part of the received signal. This effect can be avoided, if the frequency shift of the useful signal can be estimated and the receiver is adjusted accordingly. In Fig. 6, firstly, simulation results are given for unknown frequency shifts for all users and secondly, for perfect knowledge of the frequency shift for the user of interest. Figure 6 shows, that for a bit error rate of $10^{-3}$ and $\Delta \Theta_{\max } T=6 \%$ the frequency error causes a degradation of the $E_{b} / N_{0}$ of $\approx 0.6 \mathrm{~dB}$ for unknown frequency offset, whereas the degration is reduced to $\approx 0.4 \mathrm{~dB}$ for known frequency offset for the user of interest at the receiver.

\section{References}

Chang, C.-M. and Chen, K.-C.: Frequency-Domain Approach to Multiuser Detection in DS-CDMA, IEEE Comm. Letters, vol. 4, no. 11, 331-333, Nov., 2000.

Dinis, R., Lam, C. T., and Falconer, D.: Carrier Synchronization Requirements for CDMA Systems with Frequency-Domain Orthogonal Signature Sequences, ISSSTA2004, Sydney, Australia, 821-825, Sep. 2004. 
Galda, D., Rohling, H., Costa, E., Haas, H., and Schulz, E. : A Low Complexity Transmitter Structure for OFDM-FDMA Uplink Systems, VTC Spring 2002, Birmingham, UK, 1737-1741, 2002.

Goto, Y., Kawamura, T., Atarashi, H., and Sawahashi, M.: Variable Spreading and Chip Repetition Factors (VSCRF-) CDMA in Reverse Link for Broadband Wireless Access, PIMRC2003, Beijing, China, 254-259, Sep. 2003.
Sorger, U., De Broeck, I., and Schnell, M.: IFDMA - A New Spread-Spectrum Multiple-Access Scheme, ICC'98, Atlanta, Georgia, USA, Jun., 1267-1272, 1998.

Xia, P., Zhou, S., and Giannakis, G. B.: Bandwidth- and PowerEfficient Multicarrier Multiple Access, IEEE Trans. On Communications, vol. 51, no.11, 1828-1837, Nov., 2003. 